\title{
The Importance of Inflammation Markers in Heart Failure Patients With Appropriate Or Inappropriate ICD Shock
}

\author{
Sabri Seyis $^{1^{*}}$, Ozge Kurmuş ${ }^{2}$ \\ ${ }^{1}$ Department of Cardiology, Istinye University Liv Hospital, Istanbul, Turkey \\ ${ }^{2}$ Department of Cardiology, Ufuk University, Ankara, Turkey
}

"Corresponding Author: Sabri Seyis, Department of Cardiology, Istinye University Liv Hospital, Istanbul, Turkey, Tel: +90 533 2472009; E-mail: sabriseyis@yahoo.com

Received: 11 November 2017; Accepted: 22 November 2017; Published: 27 November 2017

\begin{abstract}
Background: C-reactive protein (CRP), neutrophil lymphocyte ratio (NLR), and platelet lymphocyte ratio (PLR) are associated with the prognosis of cardiovascular diseases such as coronary artery disease, acute myocardial infarction, and heart failure. However, their prognostic significance is unknown in the heart failure patients with implantable cardioverter defibrillator (ICD) devices.
\end{abstract}

Objective: The aim of this study is to evaluate the levels of inflammation markers (CRP, NLR, and PLR) in patients with ICD who have received no shock during 3 years of follow-up and in those who have received appropriate or inappropriate shock, and to determine importance of inflammatory markers in ICD patients who received shock delivery.

Results: There was a statistically significant difference between the groups in terms of gender and age ( $p=0.013$, $p<0.001$, respectively). Patients who have received appropriate or inappropriate shock were older and mostly male. There was no statistically significant difference between groups in terms of mean NLR and PLR values ( $p>0.05)$. In ICD patients who have received appropriate or inappropriate shock, the highest AUC value was found for CRP in the receiver operating characteristic curve assessment.

Conclusion: Our results show that the NLR, PLR, WBC values cannot be used to determine ICD shock while it suggests CRP may be.

Keywords: Neutrophil lymphocyte ratio; Platelet lymphocyte ratio; C-reactive protein; ICD shock 


\section{Introduction}

Implantable Cardioverter Defibrillator (ICD) therapy is a widely used type of treatment known to reduce mortality in the heart failure patients with New York Heart Association (NYHA) class II and III symptoms [1]. The most important advantage of ICD is reducing the mortality in high-risk patients with sudden cardiac arrest and cardiac disease [2]. Despite its positive effects on mortality, inappropriate ICD shocks are one of the most important complications of the treatment process [3]. They can be seen in approximately $14-29 \%$ of patients and constitutes $50 \%$ of ICD-related complications [4]. Inappropriate ICD shocks may be caused mainly by atrial fibrillation and rapid atrial arrhythmias, sinus tachycardia, lead detection problems, and noise in general. The constant prospect that the ICD will shock disturbs the quality of life by causing anxiety and stress in the patient [5].

Leukocytes, or white blood cells and subgroups, are inflammatory markers and have prognostic value in cardiovascular disease [6]. Higher neutrophil levels have been associated with mortality and poor outcome in cardiovascular diseases [7]. It has been shown in recent years that the neutrophil lymphocyte ratio (NLR) and platelet lymphocyte ratio (PLR) may be indicative of systemic inflammation and are associated with prognosis in cardiovascular diseases such as coronary artery disease, acute myocardial infarction, and heart failure, malignancies, and chronic inflammatory diseases [8]. It was also found that NLR and PLR are related with erythrocyte sedimentation rate (ESR) and C-reactive protein (CRP) [9].

Heart failure patients with reduced ejection fraction (EF) carry a risk of developing malignant ventricular arrhythmias such as ventricular tachycardia (VT) and ventricular fibrillation (VF). ICDs may prevent sudden cardiac death due to these arrhythmias. In a study by Yücel et al. involving 58 heart failure patients with ICD, higher values of NLR were shown to be a strong predictor of appropriate ICD shock [10]. Although there was one study present in the literature on appropriate ICD shock, there were no studies evaluating inappropriate ICD shocks. This study aimed to determine the relationship between inappropriate ICD shocks and markers such as CRP, white blood cell count (WBC), platelet count (Tr), neutrophil lymphocyte ratio (NLR), platelet lymphocyte ratio (PLR), mean platelet volume (MPV) in patients with ICD.

\section{Methods}

This was a retrospective study where the data were obtained by scanning the 3 -year records of 180 patients implanted with ICD due to heart failure diagnosis between 2011 and 2014. The patients were divided into two groups as ICD patients who received appropriate or inappropriate shock $(n=60)$ and patients who received no shock $(\mathrm{n}=120)$. Patients' age, gender, and laboratory parameters (CRP, WBC, MPV, Platelet, Neutrophil, and Lymphocyte) were taken from their files. Patients with severe liver and renal failure, myocardial infarction within the last 6 months, hyperthyroidism, hypothyroidism, chronic obstructive pulmonary disease, malignancy, acute or chronic infection, and systemic inflammatory rheumatic disease were excluded. The NLR value was calculated by dividing the neutrophil count to the lymphocyte count; the PLR value was calculated by dividing the platelet count to the lymphocyte count. The CRP, WBC, MPV, Tr, NLR, and PLR values of both groups were compared. The necessary approval for the study was obtained from the local ethics committee. 


\subsection{Statistical analysis}

Statistical evaluation was done using the SPSS software (version 21.0) (IBM, SPSS, Chicago, IL, USA). Numerical data were presented as mean \pm standard deviation; categorical data were presented as number and percentage. Student's $t$-test was used to compare continuous variables from two independent groups. Chi-square $\left(\chi^{2}\right)$ test was used for comparisons of categorical data from two independent groups. Statistical significance level was set at $p<0.05$. Receiver operating characteristic (ROC) curve was used to evaluate the markers' predictive power for ICD shock. The benchmark values for the definition of the best test in this method were set as follows: the sensitivity is $100 \%$, the false positivity is 0 (1Specificity $=0$ ), the accuracy (area under the curve - AUC) is 1 , and the diagnostic value of AUC is $p<0.05$.

\section{Results}

A total of 180 patients with congestive heart failure who had ICD were enrolled in the study; of these, 120 (66.7\%) had not experienced any shock during the 3-year follow-up period and $60(33.3 \%)$ had received appropriate or inappropriate shock. When the groups were evaluated in terms of gender, 94 males (78.3\%) and 26 females (21.7\%) were in the first group and 36 males (60\%) and 24 females (40\%) in the second group. The mean age was 56.88 \pm 8.91 in the first group and $66.75 \pm 8.92$ in the second group was. There was a significant difference between the groups when compared by gender and age ( $p=0.013, p<0.001$, respectively). Groups were compared for the presence of hypertension, diabetes mellitus, and hyperlipidemia. Incidence of hypertension and diabetes mellitus was significantly higher in the patient group that had received shock than the patient group that had not received shock $(p<0.001)$. The CRP, WBC, Tr, MPV, NLR, and PLR values of the patients were examined. The clinical characteristics of the patients and the laboratory results are presented in Table 1. The mean NLR value was 4.38 \pm 1.88 in the group that had not received shock and $4.08 \pm 1.61$ in the group that had received shock. The mean PLR value was $186.33 \pm 53.95$ in the group that had not received shock and $182.68 \pm 47.75$ in the group that had received shock. There was no significant difference between groups in terms of mean NLR and PLR values $(p>0.05)$.

When the markers were evaluated for their predictive power, the highest $\mathrm{AUC}$ value was found for CRP ( $\mathrm{AUC}=0.875$ ), which was followed by WBC ( $\mathrm{AUC}=0.516$ ), NLR (AUC=0.534), PLR (AUC=0.504), platelet count (AUC $=0.554)$, and MPV (AUC $=0.537)$. Sensitivity and specificity were determined as $71.6 \%$ and $89.1 \%(p<0.001)$, respectively, when a cutoff value of 0.3 was used for CRP in ROC analysis applied for appropriate or inappropriate ICD shock. The cutoff, sensitivity, specificity, AUC, 95\% confidence interval, and $p$ values for the ROC analysis of predictive powers of CRP, WBC, Tr, MPV, NLR, and PLR values for predicting the ICD patients that do and do not receive ICD shock are given in Table 2.

\begin{tabular}{|l|l|l|l|}
\hline & $\begin{array}{l}\text { No shock } \\
(\mathbf{n = 1 2 0})\end{array}$ & $\begin{array}{l}\text { Shock received } \\
(\mathbf{n = 6 0 )}\end{array}$ & $\boldsymbol{p}$ value \\
\hline Age, year & $56.88 \pm 8.91$ & $66.75 \pm 8.92$ & $<\mathbf{0 . 0 0 1}$ \\
\hline Gender $(\mathrm{M} / \mathrm{F}), \mathrm{n}(\%)$ & $94(78.3) / 26(21.7)$ & $36(60) / 24(40)$ & $\mathbf{0 . 0 1 3}$ \\
\hline BMI, $\mathrm{kg} / \mathrm{m}^{2}$ & $27.08 \pm 2.58$ & $26.80 \pm 2.43$ & 0.481 \\
\hline
\end{tabular}




\begin{tabular}{|l|l|l|l|}
\hline Diabetes Mellitus, $\mathrm{n}(\%)$ & $42(35)$ & $45(75)$ & $<\mathbf{0 . 0 0 1}$ \\
\hline Hypertension, $\mathrm{n}(\%)$ & $55(45.8)$ & $56(93.3)$ & $<\mathbf{0 . 0 0 1}$ \\
\hline Hyperlipidemia, $\mathrm{n}(\%)$ & $50(41.7)$ & $26(43.3)$ & 0.874 \\
\hline WBC, $\mathrm{x} 10^{3}$ & $7.66 \pm 1.76$ & $7.83 \pm 1.81$ & 0.547 \\
\hline CRP, $\mathrm{mg} / \mathrm{dL}$ & $0.21 \pm 0.08$ & $0.40 \pm 0.12$ & $<\mathbf{0 . 0 0 1}$ \\
\hline Platelet, $\mathrm{x} 10^{3}$ & $255 \pm 63$ & $255 \pm 88$ & 0.951 \\
\hline MPV, fl & $10.73 \pm 0.87$ & $10.84 \pm 0.91$ & 0.461 \\
\hline NLR & $4.38 \pm 1.88$ & $4.08 \pm 1.61$ & 0.294 \\
\hline PLR & $186.33 \pm 53.95$ & $182.68 \pm 47.75$ & 0.658 \\
\hline
\end{tabular}

Table 1: The distribution of the clinical characteristics and the laboratory results of the patients with ICD

Data are presented as mean \pm standard deviation or number of patients (percent).

BMI: Body mass index; WBC: White blood cell; CRP: C-reactive protein; MPV: Mean platelet volume; NLR:

Neutrophil lymphocyte ratio; PLR: Platelet lymphocyte ratio; n: Number of patients.

The $p$ values $<0.05$ were considered statistically significant.

\begin{tabular}{|l|l|l|l|l|l|l|}
\hline & AUC & Sensitivity & Specificity & Cutoff & $\mathbf{9 5 \%}$ CI & $p$ \\
\hline CRP & $\mathbf{0 . 8 9 2}$ & $\mathbf{7 1 . 6 7}$ & $\mathbf{8 9 . 1 7}$ & $>\mathbf{0 . 3}$ & $\mathbf{0 . 8 3 7}$ to $\mathbf{0 . 9 3 3}$ & $<\mathbf{0 . 0 0 0 1}$ \\
\hline MPV & 0.537 & 68,33 & 45 & $>10.5$ & 0.462 to 0.612 & 0.4168 \\
\hline Platelet & 0.554 & 38,33 & 79.17 & $\leq 207000$ & 0.479 to 0.628 & 0.2667 \\
\hline WBC & 0.516 & 88,33 & 18.33 & $>5600$ & 0.441 to 0.591 & 0.7171 \\
\hline PLR & 0.504 & 8,33 & 81.67 & $>255$ & 0.429 to 0.579 & 0.9238 \\
\hline NLR & 0.534 & 85 & 27.5 & $\leq 5.6$ & 0.458 to 0.608 & 0.4498 \\
\hline
\end{tabular}

Table 2: The cutoff, sensitivity, specificity, AUC, $95 \%$ confidence interval, and $p$ values for the ROC analysis of predictive powers of CRP, WBC, Tr, MPV, NLR, and PLR values for receiving appropriate or inappropriate ICD shock.

Receiver operating characteristic (ROC) curve was used to calculate these values for the groups.

CRP: C-reactive protein; WBC: White blood cell; MPV: Mean platelet volume; NLR: Neutrophil lymphocyte ratio; PLR: Platelet lymphocyte ratio; AUC: Area under the curve; CI: Confidence interval.

The $p$ values $<0.05$ were considered statistically significant.

\section{Discussion}

This is the first study to evaluate the association between appropriate or inappropriate shocks and inflammatory markers in patients implanted with ICD due to heart failure. We have shown in this study that there is a relationship 
between high CRP values and ICD shock delivery. Our results suggested that NLR and PLR values are not related to appropriate or inappropriate ICD shock delivery.

Heart failure is a disease that causes significant morbidity and mortality. More than $50 \%$ of patients with heart failure die of dysrhythmia, primarily of ventricular tachyarrhythmia [11]. ICD therapy is a treatment method that reduces mortality in patients with sudden cardiac arrest and in high-risk patients with heart disease successfully applied to prevent ventricular arrhythmias [2]. Inappropriate ICD shocks are one of the most important complications and make the treatment process difficult for patients [3]. The most common causes for inappropriate ICD shocks are atrial fibrillation, supraventricular tachycardia, T-wave oversensing, lead malfunctions, and noise [5]. There is no study in the literature investigating the relationship between the delivery of appropriate or inappropriate ICD shocks and inflammatory markers. Our study has shown that patients who received appropriate or inappropriate shock during the 3-year follow-up of ICD implantation were older, mostly males, and had a higher incidence of hypertension and diabetes mellitus.

Neutrophils, lymphocytes, and platelets are the blood cells involved in the inflammatory process. NLR and PLR values are easily calculable and quite low-cost tests; the use of NLR is suggested as a new marker for systemic inflammation [12]. It has been observed that the NLR value may help in determining mortality in acute coronary syndrome and high NLR values are associated with increased mortality [12]. The PLR value is also an inflammatory marker as NLR and is used to indicate chronic inflammation. Azab et al. have shown that high PLR values increase mortality in patients who had myocardial infarction [13]. In another study, PLR was shown to be a better predictor of inflammation than NLR in patients with end-stage renal failure [14].

Although high WBC, PLR, and NLR values were reported in literature to be useful as prognostic markers for cardiovascular disease and inflammation, we have found that these markers are not sufficient to predict appropriate or inappropriate ICD shocks $[8,13,14]$. Conversely, we have found that high CRP levels can be used as a better indicator for appropriate or inappropriate ICD shock, with $71.6 \%$ sensitivity and $89.1 \%$ specificity.

There are studies in the literature showing that inflammatory pathways may play a central role in the development of heart failure. An association between inflammatory cytokines such as CRP, interleukin-6, and TNF- $\alpha$ and the incidence of heart failure has been shown in these studies [15,16]. Although there is no correlation between NLR, PLR, and WBC levels and shock delivery in our study, it can be said that CRP may be related to shock delivery. Possible reason for this may be that age, hypertension, presence of diabetes mellitus, and elevated levels of CRP have an arrhythmogenic effect and may induce ICD shock delivery.

Our work has some limitations, the most important one being that it is a retrospective study and there were no control group. The lack of use of additional laboratory markers other than hemogram and CRP is another limitation.

\section{References}

1. Connolly SJ, Hallstrom AP, Cappato R, et al. Meta-analysis of the implantable cardioverter defibrillator 
secondary prevention trials. Eur Heart J 21 (2000): 2071-2078.

2. Cevik C, Perez-Verdia A, Nugent K. Implantable cardioverter defibrillators and their role in heart failure progression. Europace 11 (2009): 710-715.

3. Oylumlu M, Oylumlu M, Ertaş F, et al. İmplante edilen kardiyoverter defibrilatörlerin uygunsuz şokunun nadir bir nedeni: Tremor. Koşuyolu Heart Journal 16 (2013): 150-152

4. Nanthakumar K, Paquette M, Newman D, Deno DC, Malden L, Gunderson B, et al. Inappropriate therapy from atrial fi brillation and sinus tachycardia in automated implantable cardioverter defibrillators. Am Heart J 139 (2000): 797-803.

5. Carroll DL, Hamilton GA. Long-term effects of implanted cardioverter defibrillators on health status, quality of life, and psychological state. Am J Crit Care 17 (2008): 222-230.

6. Horne BD, Anderson JL, John JM, Weaver A, Bair TL, Jensen KR, et al. Which white blood cell subtypes predict increased cardiovascular risk? J Am Coll Cardiol 45 (2005): 1638-1643.

7. Uthamalingam S, Patvardhan EA, Subramanian S, Ahmed W, Martin W, Daley M, et al. Utility of the neutrophil to lymphocyte ratio in predicting long-term outcomes in acute decompensated heart failure. Am J Cardiol 107 (2011): 433-438.

8. Balta S, Demirkol S, Unlu M, Arslan Z, Celik T. Neutrophil to lymphocyte ratio may be predict of mortality in all conditions. Br J Cancer 109 (2013): 3125-3126.

9. Peng YF, Cao L, Zeng YH, Zhang ZX, Chen D, Zhang Q, Zhu YS. Platelet to lymphocyte ratio and neutrophil to lymphocyte ratio in patients with rheumatoid arthritis. Open Med 10 (2015): 249-253.

10. Yucel H, Zorlu A, Kaya H, Kıvrak T, Kurt R, Yıldırımlı K, Yilmaz MB. Elevated Neutrophil/Lymphocyte Ratio is Associated with Appropriate Shock After ICD Implantation. J Clin Anal Med 7 (2016): 772-776.

11. Fogoros RN, Elson JJ, Bonnet CA, Fiedler SB, Burkholder JA. Efficacy of the automatic implantable cardioverter-defibrillator in prolonging survival in patients with severe underlying cardiac disease. $\mathrm{J}$ Am Coll Cardiol 16 (1990): 381-386.

12. Bhat T, Teli S, Rijal J, Bhat H, Raza M, Khoueiry G, et al. Neutrophil to lymphocyte ratio and cardiovascular diseases: a review. Expert Rev Cardiovasc Ther 11 (2013): 55-59.

13. Azab B, Shah N, Akerman M, McGinn JT Jr. Value of platelet/lymphocyte ratio as a predictor of allcause mortality after non-ST-elevation myocardial infarction. J Thromb Thrombolysis 34 (2012): 326-334.

14. Turkmen K. Platelet-to-Lymphocyte Ratio: One of the novel and valuable platelet indices in hemodialysis patients. Hemodial Int 17 (2013): 670.

15. Marshe VS, Pira S, Mantere O, Bosche B, Looper KJ, Herrmann N, Müller DJ, Rej S. C-reactive protein and cardiovascular risk in bipolar disorder patients: A systematic review. Prog Neuropsychopharmacol Biol Psychiatry 79 (2017): 442-451.

16. Ohkuma T, Jun M, Woodward M, Zoungas S, Cooper M, et al. Advance Collaborative Group. Cardiac Stress and Inflammatory Markers as Predictors of Heart Failure in Patients With Type 2 Diabetes: The ADVANCE Trial. Diabetes Care 40 (2017): 1203-1209.

This article is an open access article distributed under the terms and conditions of the

$\underline{\text { Creative Commons Attribution (CC-BY) license } 4.0}$ 